\title{
Scattering by a Potential Using Hyperbolic Methods
}

\author{
By Alvin Bayliss, Yanyan Li, and Cathleen S. Morawetz* \\ Dedicated to Eugene Isaacson on his 70th birthday
}

\begin{abstract}
This is a preliminary note on a numerical time-dependent approach to the inverse scattering problem where the data given is back scattering. The results in one space variable are both computed and are quite comparable to the best existing results. A corresponding algorithm is developed for two variables. A major stage is the computation of the forward problem for the wave equation with potential and with an incoming plane wave. Interesting developments are a boundary layer next to the propagating delta function and effective resolution of the scattered wave. It is assumed there are no bound states.
\end{abstract}

In one-dimensional scattering many methods have been developed to determine $q(x)$ on $-\infty<x<+\infty$ from data, both prescribed and collected at infinity, on solutions of

$$
u_{x x}-q(x) u=\lambda u
$$

for appropriate ranges of $\lambda$. In higher numbers of space variables not much is known, and many of the methods that work well in one space variable cannot be carried over.

This is a preliminary note on a numerical time-dependent approach to the problem. An algorithm is developed for an arbitrary number of dimensions for the backscattering problem, Section 1. The results in one space variable are both computed and analyzed, Section 2. The algorithm and differences when one goes to more than one space variable are described in Section 3; in particular, the development of a boundary layer when a plane wave impinges is described and analyzed in Section 4. In Section 5 computations of the forward problem for two space variables are described with special emphasis on the nature of the boundary layer as well as the prospective role of these computations in the two-dimensional back-scattering problem.

It is assumed there are no bound states.

1. Integral Equation for $q$ in Terms of Back-Scattered Data. The inverse back-scattering problem is usually viewed, see for example [7], in the following way.

Received March 21, 1988.

1980 Mathematics Subject Classification (1985 Revision). Primary 35R30, 65P05; Secondary 65M99, 65N99, 81-04, 81F99.

Key words and phrases. Scattering from a potential, inverse problems.

*This manuscript has been authored under contract number N00014-85-K-0195 with the U.S. Department of the Navy, Office of Naval Research and supported in part by the National Science Foundation under grant number (312)491-7221-DMS-8701543. Some computations were done on the Cray at the Naval Research Laboratory under contract number F49620-87-C-0065. Accordingly, the U.S. Government retains a nonexclusive, royalty-free license to publish or reproduce the published form of this contribution, or allow others to do so, for U.S. Government purposes. 
Let $p$ be the solution in $R^{n}$ of

$$
\Delta p+\left(\omega^{2}-q(x)\right) p=0
$$

which as $|x| \rightarrow \infty, x=-e|x|$ behaves like

$$
\exp (-i \omega e \cdot x)+s(\omega, e)|x|^{-(n-1) / 2} \exp (-i \omega|x|)
$$

Here, $e$ is a unit vector. The inverse problem is to determine $q(x)$, given $s(\omega, e)$ for all real values of $\omega$ and $e$ over a sphere.

We view the problem as a hyperbolic problem by considering $P(e, t, x)$ to be the solution of

$$
U_{t t}-\Delta U+q U=0
$$

which as $t \rightarrow-\infty$ becomes $\delta(t-e \cdot x)$. If $q$ has compact support, then $P \equiv \delta(t-e \cdot x)$ for $t<T_{0}(e)$. This can also be generalized for $q$ decaying sufficiently rapidly at $\infty$ but we omit this here. We assume there are no bound states, for otherwise $P \rightarrow \infty$ as $t \rightarrow \infty$.

If we take the Fourier transform in time we find that the $p$ given above is

$$
\int_{-\infty}^{+\infty} e^{i \omega t} P(e, t, x) d t
$$

As $t \rightarrow+\infty, t-e \cdot x>0$, one can show that for $t-e \cdot x \geq \varepsilon>0$

$$
P \rightarrow S\left(e, t-|x|, e^{\prime}\right)|x|^{-(n-1) / 2}
$$

where $e^{\prime}=x /|x|$. The back-scattering problem is to find $q$, given $S(e, t-|x|,-e)$.

There is an integral equation which relates $S(e, s,-e)$, with $s=t-|x|$, to $q$ through the solutions $P$ for all angles. It involves a Radon transform for $n \geq 2$. We prove first a theorem relating averages of $q$ to the back-scattering. We shall henceforth for convenience assume that $q$ has compact support.

Basic relation. Let $C_{n}=\int d \Omega^{n-1}$. Then

$$
\begin{aligned}
\int_{x_{\perp} \cdot e=0} q\left(x+x_{\perp}\right)\left|d x_{\perp}\right|= & -2 C_{n} \int_{0}^{\infty} S_{s}\left(e, 2 e \cdot x-\frac{1}{2} \tau^{2}, e\right) \tau^{n-2} d \tau \\
& -2 \int_{e \cdot x^{\prime}<e \cdot x} P_{\text {scat }}\left(e, 2 e \cdot\left(x-x^{\prime}\right), x^{\prime}\right) q\left(x^{\prime}\right)\left|d x^{\prime}\right|
\end{aligned}
$$

where $P_{\text {scat }}$ is defined below.

To prove this relation, we make use of certain properties of $P(e, t, x)$.

First of all, we should regard the condition

$$
P=\delta(t-e \cdot x)
$$

for $t<T_{0}(e)$ as an initial condition. Hence, by a domain of dependence argument one has

$$
P \equiv 0 \text { for } t-e \cdot x<0 \text {. }
$$

We write $P$ as $\delta(t-e \cdot x)+P_{\text {scat }}(e, t, x)$, and by propagation of singularities theory one finds

$$
P_{\mathrm{scat}}=-\frac{1}{2}\left(\int_{-\infty}^{e \cdot x} q\left(x_{0}+s e\right) d s\right) H(t-e \cdot x)+C
$$


with $e \cdot x_{0}=-\infty$ and $x_{0}$ parallel to $x$. This holds for $t-e \cdot x<t_{0}$, say. Here, $C$ is continuous across $t=e \cdot x$ and $H$ is a Heaviside function.

These properties of $P$ are reflected in $p$ by analytic continuation properties and asymptotic behavior for large $|\omega|, \operatorname{Im} \omega<0$.

Of course, (1.2) gives the behavior of $P_{\text {scat }}$ for large values of $t$. We note that we assume

$$
S \text { and its derivatives tend to zero as } t-|x| \rightarrow \infty \text {. }
$$

Proof of Basic Relation. Let $e_{0}=(1,0, \ldots)$ and let $U=P\left(e_{0}, t, x\right), V=$ $P\left(e_{0}, s-t, x\right)$. Then from $\left(U_{t} V-V_{t} U_{t}\right)_{t}-\operatorname{div}(V \nabla U-U \nabla V)=0$ integrated over the wedge $t \leq T, t-x_{1} \geq 0+, x_{1}<T$ we have for $T>s / 2$

$$
\int_{\substack{t=T \\ x_{1}<T}}\left(U_{t} V-V_{t} U\right)|d x|=2 \int_{\substack{t-x_{1}=0 \\ t<T}} V\left(U_{t}+U_{x_{1}}\right)|d x| .
$$

Note there is no contribution from $x_{1}=-\infty$ since $P=\delta\left(t-e_{0} \cdot x\right)$. The righthand side is obtained by integrating by parts. The boundary term vanishes because $V=P\left(e_{0}, s-T, x\right)$ is identically zero for $s-T-e_{0} \cdot x<0,(1.3)$.

Next, let $T$ be sufficiently large so that $V=P\left(e_{0}, s-t, x\right) \equiv \delta\left(s-t-e_{0} \cdot x\right)$ for $t=T$. Then the left-hand side of (1.6) is

$$
\begin{gathered}
\int_{t=T}\left(P_{t}\left(e_{0}, t, x\right) \delta\left(s-t-x_{1}\right)-P\left(e_{0}, t, x\right) \delta_{t}\left(s-t-x_{1}\right)\right)|d x| \\
=\int\left(P_{t}\left(e_{0}, s-T, \xi\right)+P_{x}\left(e_{0}, s-T, \xi\right)\right)\left|d x_{\perp}\right|
\end{gathered}
$$

where $x_{\perp}$ is the $(n-1)$-dimensional vector $\left(x_{2}, x_{3}, \ldots, x_{n}\right)$ and $\xi=\left(e-T, x_{\perp}\right)$.

For $n=1$ we have simply $P_{t}(T, s-T)+P_{x}(T, s-T)$. The right-hand side of (1.6), by (1.4), is

$$
-\int_{x_{1} \leq T} P\left(e_{0}, s-x_{1}, x\right) q(x)|d x| .
$$

We now let $T \rightarrow \infty$. In the left-hand side for $n>1$ we may replace $P$ by its scattered data $(1.2)$ since $T-e_{0} \cdot \xi=2 T-s \rightarrow \infty$. This yields

$$
\int \frac{S^{\prime}\left(e_{0}, T-|\xi|, \xi /|\xi|\right)}{|\xi|^{(n-1) / 2}}\left(1-\frac{s-T}{|\xi|}\right)\left|d x_{\perp}\right| .
$$

Here, prime denotes the derivative with respect to the second argument. Let $T-$ $s /|\xi|=\cos \theta$. Then $\sin \theta=\left|x_{\perp}\right| /|\xi|$ and

$$
T-|\xi|=T(1-\sec \theta)+s \sec \theta .
$$

By (1.5), $S^{\prime} \rightarrow 0$ when its middle argument goes to infinity. So the main contribution is from $\theta=0$. There, the integrand tends to

$$
2 \frac{S^{\prime}\left(e_{0}, s-\frac{1}{2} T \theta^{2},-e_{0}\right)}{(T-s)^{(n-1) / 2}}
$$

and $\left|d x_{\perp}\right|=\left|x_{\perp}\right|^{n-2} d\left|x_{\perp}\right| d \Omega^{n-1}$, where $\Omega^{n-1}$ is a solid angle on the unit sphere in $R^{n-1}$, and $\left|x_{\perp}\right|^{n-2} d\left|x_{\perp}\right| \sim(T-s)^{n-1} \sin ^{n-2} \theta d \sin \theta \sim(T-s)^{n-1} \theta^{n-2} d \theta$. Setting $T^{1 / 2} \theta=\tau$, we see that the integral tends to

$$
2 \int_{0}^{\infty} S^{\prime}\left(e_{0}, s-\frac{1}{2} \tau^{2},-e_{0}\right) \tau^{n-2} d \tau d \Omega^{n-1}
$$


The remainders are easily shown to be of lower order as $T \rightarrow \infty$ and hence vanish.

Inserting this limit in the identity along with (1.5) we obtain, with $C_{n}=$ $\int d \Omega^{n-1}$

$$
\begin{aligned}
2 C_{n} & \int_{0}^{\infty} S^{\prime}\left(e_{0}, s-\frac{1}{2} \tau^{2},-e_{0}\right) \tau^{n-2} d \tau \\
& =-\int_{x_{1} \leq \infty} P\left(e_{0}, s-x_{1}, x\right) q(x)|d x| .
\end{aligned}
$$

This is easily reduced to the basic relation.

The formula can also be obtained directly by applying the fundamental solution of the wave equation tom $P=q P$.

Since the right-hand side may be rewritten as

$$
\int\left(\delta\left(s-2 x_{1}\right)+P_{\text {scat }}\left(e_{0}, s-x_{1}, x\right)\right) q(x)|d x|,
$$

we have that

$$
\int q\left(\frac{s}{2} e_{0}+x_{\perp}\right)\left|d x_{\perp}\right|+2 \int P_{\text {scat }}\left(e_{0}, s-x_{1}, x\right) q(x)|d x|
$$

is given in terms of the scattering data. Since the second term is quadratic in $q$, we may rewrite this as

$$
\begin{gathered}
\int\left(q\left(\frac{s}{2} e_{0}+x_{\perp}\right)-q_{B}\left(\frac{s}{2} e_{0}+x_{\perp}\right)\right)\left|d x_{\perp}\right| \\
+2 \int P_{\text {scat }}\left(e_{0}, s-x_{1}, x\right) q(x)|d x|=0
\end{gathered}
$$

where $q_{B}$ is the Born approximation or linearized approximation for $q$.

Changing to general coordinates, we have

$$
\begin{aligned}
& \int\left(q\left(s e+x_{\perp}\right)-q_{B}\left(s e+x_{\perp}\right)\left|d x_{\perp}\right|\right. \\
& +2 \int P_{\text {scat }}(e, 2 s-e \cdot x, x) q(x)|d x|=0 .
\end{aligned}
$$

Letting the Radon transform

$$
\text { R.T. } f(x)=\int f\left(s e+x_{\perp}\right)\left|d x_{\perp}\right|
$$

one gets

$$
q(x)-q_{B}(x)=2(\mathrm{R} . \mathrm{T} .)^{-1} \int P_{\mathrm{scat}}\left(e, 2 s-e \cdot x^{\prime}, x^{\prime}\right) q\left(x^{\prime}\right)\left|d x^{\prime}\right| .
$$

2. The One-Dimensional Case. For $n=1$, outside of the support of $q$, $P=S(t+x)$ and the left-hand side of the equation is $2 S^{\prime}(s)$. Thus

$$
q(x)=-2 S_{s}(x)-\int_{x^{\prime}<x} P_{\text {scat }}\left(2 x-x^{\prime}, x^{\prime}\right) q(x) d x .
$$

In the one-dimensional case there are many methods for solving the inverse problem. Numerically, the best methods are those that take advantage of the fact that $q(x)$ is determined by the values of $S(s)$ for $s<2 x$. The methods of Bube and Burridge [1] are extremely accurate. They change variables but essentially use, 
for determining $q$, the expression (1.4) and solve an equation like the hyperbolic equation (1.1) from left to right. Deift and Trubowitz [3] solve the problem in the Fourier transform plane also proceeding from left to right, see also Stickler [8]. In both cases, the values of $q(x)$ for large $x$ are harder to compute and require extreme accuracy in computing $q$ for small $x$. The approach used here is an extension of the previous work of Kriegsmann and Morawetz [6].

The basic relation provides us with an algorithm for determining $q(x)$. We note that it is a Volterra-like equation and that the values of $P$ involve only the values of $q$ up to $x$.

The algorithm for combining is: take a trial $q$, compute $P$, insert $P q$ in the right-hand side of the basic relation and obtain a new value of $q$. Thus, at the $n$th iteration,

$$
q_{n}=-2 S_{s}(x)-2 \int_{x^{\prime}<x} P_{n-1, \mathrm{scat}}\left(2 x-x^{\prime}, x^{\prime}\right) q_{n-1}\left(x^{\prime}\right) d x^{\prime}
$$

where $P_{n-1, \text { scat }}$ is the numerical solution of (1.1) with the data

$$
\begin{aligned}
& q=q_{n-1}(x), \\
& P_{n-1, \text { scat }}(x, x)=-\frac{1}{2} \int_{-\infty}^{x} q_{n-1}\left(x^{\prime}\right) d x^{\prime}
\end{aligned}
$$

This works very well over a range of $x$. The numerical solution was obtained in two ways: (a) in $x, t$ coordinates with a $t$-mesh half the size of the $x$-mesh and the data near $t=x$ fitted by averaging and (b) in characteristic coordinates, using a stable method for Goursat problems. The first iteration began with $q=0$, and therefore $q_{1}$ is the Born approximation. In the cases of interest, $q_{1}$ is very different from $q$.

Details of the method are described below and a typical result is given in Figures 2.1 and 2.2. Our objective was to get the best results on a coarse mesh because to carry out the corresponding algorithm in two space dimensions we must iterate on functions of four variables such as $P(e, t, x)$. From the basic relation one can easily see that there are no simple domain of dependence arguments, and since our objective is to do a two-dimensional computation, we tried to avoid using the domain of dependence. However, if the range of $x$ was large compared to the support of $q$, then the values of $q$ near the end of the support failed to converge.

Remark. To check out the algorithm in another way, the computations by method (b) were also carried out in steps, successively accepting a value of $q$ up to say $x_{1}$ after several iterations and then finding $q$ from $x_{1}$ to $x_{1}+\Delta x$ using instead of $(2.1)$

$$
\begin{aligned}
q_{n}= & q^{*} \quad \text { for } x<x_{1}, \\
q_{n}= & -2\left(S_{s}(2 x)-S_{s}^{*}(2 x)\right) \\
& -2 \int_{x^{\prime}<x}\left(P_{n-1, \text { scat }}\left(2 x-x^{\prime}, x^{\prime}\right) q_{n-1}\left(x^{\prime}\right)\right. \\
& \left.-P_{\text {scat }}^{*}\left(2 x-x^{\prime}, x^{\prime}\right) q^{*}\left(x^{\prime}\right)\right) d x^{\prime} \quad \text { for } x_{1}<x<x_{1}+\Delta x,
\end{aligned}
$$




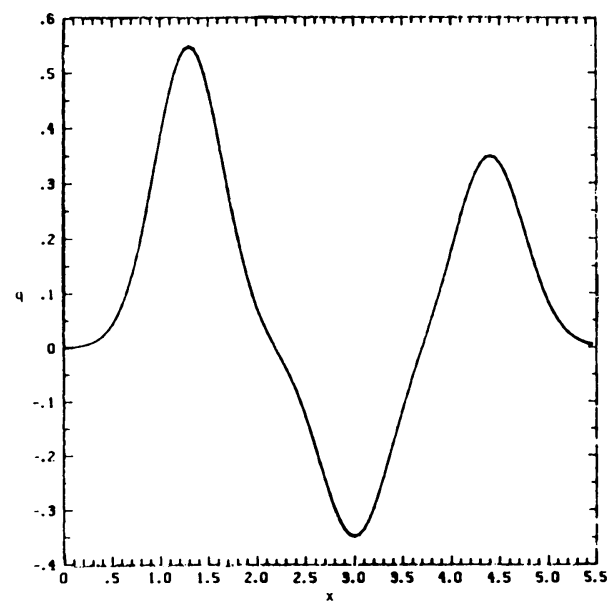

FIGURE 2.1

Here $q=Q_{1} \exp \left(-4\left(x-C_{1}\right)^{2}\right)+Q_{2} \exp \left(-4\left(x-C_{2}\right)^{2}\right)$ $+Q_{3} \exp \left(4-\left(x-C_{3}\right)^{2}\right)$ with $Q_{1}=0.55, Q_{2}=-0.35, Q_{3}=0.35$, $C_{1}=1.3, C_{2}=3.00$ and $C_{3}=4.4$. The mesh size is 0.05 .

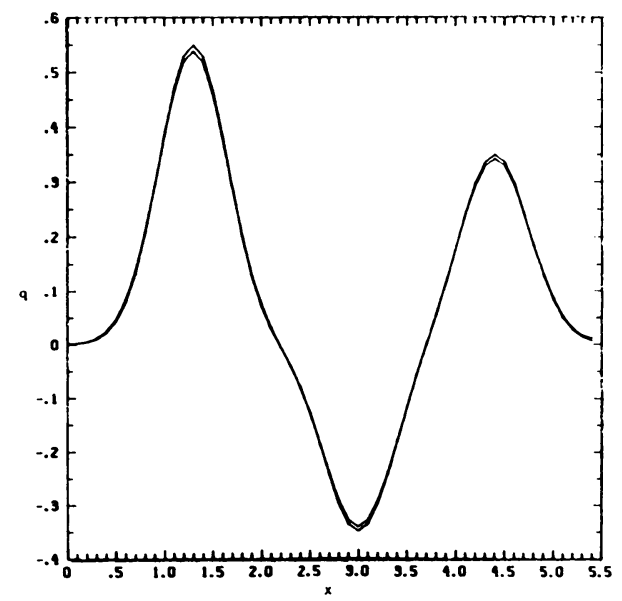

FIGURE 2.2

As in Figure 2.1 with mesh size $=1$.

where $q^{*}$ is the "accepted" value of $q$ for $x<x_{1}$, and 0 for $x>x_{1} ; S^{*}$ is the scattered field for $q^{*}$ and $P_{\mathrm{scat}}^{*}$ is the scattered wave. Hence, for $x<x_{1}, P_{\text {scat }}-P_{\mathrm{scat}}^{*}$ and

$$
q^{*} \sim-2 S_{s}(2 x)-2 \int_{x^{\prime}<x} P_{\text {scat }}\left(2 x-x^{\prime}, x^{\prime}\right) q^{*} d x^{\prime},
$$

where $\sim$ means to some prescribed accuracy. By the basic relation, for $x>x_{1}$,

$$
\begin{aligned}
q \sim & -2\left(S_{s}(2 x)-S_{s}^{*}(2 x)\right) \\
& -\int_{x^{\prime}<x}\left(P_{\text {scat }}\left(2 x-x^{\prime}, x^{\prime}\right) q\left(x^{\prime}\right)-P_{\text {scat }}^{*}\left(2 x-x^{\prime}, x^{\prime}\right) q^{*}\left(x^{\prime}\right)\right) d x^{\prime} .
\end{aligned}
$$

This justifies (2.1a) and the iteration will converge for $\Delta x$ small enough. 
The results using this algorithm were undistinguishable from the previous ones.

Remark. If in iterating on the equation of the Basic Relation we are close to the situation that the main contribution to $P_{\text {scat }}$ comes from the coefficient of the Heaviside function, then the equation tends to look like

$$
q(x)=-2 S_{s}(2 x)+\int^{x}\left(\int^{x^{\prime}} q\left(x^{\prime \prime}\right) d x^{\prime \prime}\right) H\left(2\left(x-x^{\prime}\right)\right) q\left(x^{\prime}\right) d x^{\prime}
$$

or with $q(x)=\dot{h}$,

$$
\dot{h}=-2 S_{s}(2 x)+\int^{x} h\left(x^{\prime}\right) \dot{h}\left(x^{\prime}\right) d x^{\prime}=-2 S_{s}(2 x)+\frac{1}{2} h^{2}(x) .
$$

This Riccati equation may not have a bounded solution. If we come near to this situation, the iteration will not work.

A final feature about the one-dimensional problem is that there is a complete analogue between the impedance problem (variable sound speed) and the potential problem. The necessary change of variable is described in [1] or [3]. This is not true in more space variables. A solution to a wave equation with a variable speed of propagation which behaves like a delta function for $t=-\infty$ will for some directions develop caustics in its singular surface.

3. Higher Dimensions. In two space dimensions, in which we want to compute, the formula (1.10) becomes

$$
q(x)-q_{B}(x)=\frac{1}{2 \pi} \int_{-\pi}^{+\pi} \int_{-\infty}^{+\pi} \frac{d s d \theta}{s} \frac{\partial}{\partial s} F(2 e \cdot x+2 s, e)
$$

where $F(s, e)=2 \int P_{\text {scat }}\left(e, s-e \cdot x^{\prime}, x^{\prime}\right) q\left(x^{\prime}\right)\left|d x^{\prime}\right|$.

The iterative algorithm to do this is: compute $P_{\text {scat }}$ using a trial $q$, integrate to find $F(s, e)$ and obtain a new $q$ from (3.1). The uniqueness and existence is proved in [2].

For $n \geq 3$ we should be dealing with (1.1) and the Basic Relations of Section 1. We refer the reader to the work of Fadeev [4], Newton [5] and Prosser [7] for other approaches and the case $n \geq 3$. In [1] there is another hyperbolic approach. Uniqueness and existence under certain conditions where the scattered data is sufficiently small has been established by Prosser [7].

4. The Direct Problem and Its Boundary Layer. In Eq. (1.4) we have the description of the solution $P_{\text {scat }}$ near the plane $t=e \cdot x$. This expression was used only locally and is actually valid only in a neighborhood of $t=e \cdot x$. For large values of $e \cdot x$, the solution drops away very rapidly to zero as $t-e \cdot x$ increases. This was first found computationally but can best be described analytically.

Let the support of $q$ lie inside $|x|=a$. Let $e$ be $(1,0, \ldots)$ for convenience. Then $P_{\text {scat }}$ satisfies the equation $m P_{\text {scat }}=-q P_{\text {scat }}$ for $t-x_{1}>0, P_{\text {scat }}=$ $-\frac{1}{2} \int_{-\infty}^{x_{1}} q\left(s, x_{\perp}\right) d s$ for $t=x_{1}$, where $q(x)=q\left(x_{1}, x_{\perp}\right)$.

Then

$$
P_{\text {scat }}=P_{1 \text { scat }}+P_{2 \text { scat }}
$$


where $P_{1 \text { scat }}$ satisfies $س P_{1 \mathrm{scat}}=-q P_{\text {scat }}$ in $R^{n} \times(-\infty,+\infty)$ and $P_{2 \text { scat }}$ satisfies $\mathrm{m} P_{2 \text { scat }}=-q \delta\left(t-s_{1}\right) . \quad P_{1 \text { scat }} \equiv 0$ for $t \rightarrow-\infty$ and for $t \rightarrow+\infty$ satisfies the radiation condition for large $|x|$.

We claim that $P_{2 s c a t}$ behaves like a function of $t\left(t-x_{\perp}\right)$ and $x_{\perp}$ as $t$ (or $x_{1}$ ) $\rightarrow \infty, t-x_{1}$ bounded.

We give the argument using the equation for $P_{\text {scat }}=F$ and suppose there are three space variables. Thus,

$$
F_{t t}-\Delta F=-q \delta\left(t-x_{1}\right)
$$

Then

$$
F=\frac{1}{4 \pi} \int_{r \leq t} \frac{\delta\left(t-x_{1}^{\prime}-r\right)}{r} q\left(x^{\prime}\right)\left|d x^{\prime}\right|
$$

with $r=\left|x-x^{\prime}\right|$. Let $t-x_{1}^{\prime}-r=s, x_{2}^{\prime}=\rho \cos \theta, x_{3}^{\prime}=\rho \sin \theta$. Then

$$
F=\frac{1}{4 \pi} \int \frac{\delta(s)}{\left(\left(x_{1}-x_{1}^{\prime}\right)^{2}+\rho^{2}\right)^{1 / 2}}\left(s_{x_{1}}\right)^{-1} q\left(x_{1}^{\prime}, \rho \cos \theta, \rho \sin \theta\right) \rho d \rho d \theta d s,
$$

where we have taken $x_{2}=x_{3}=0$ for case of proof. Integrating the delta function, we have with $x_{1}^{\prime}=\xi(s, \rho)$,

$$
F=-\frac{1}{4 \pi} \int \frac{q(\xi(0, \rho), \rho \cos \theta, \rho \sin \theta)}{\left(\left(x_{1}-\xi\right)^{2}+\rho^{2}\right)^{1 / 2}-(x-\xi)} \rho d \rho d \theta
$$

and $\xi+\sqrt{(x-\xi)^{2}+\rho^{2}}=t$ for $s=0$, i.e., $\xi=\frac{1}{2}(t+x)-\rho^{2} / 2(t-x)$. We set $X=\frac{1}{2}\left(t+x-\rho^{2} /(t-x)\right)$ and note that $q$ has compact support:

$$
\begin{array}{r}
F=\frac{-1}{4 \pi} \int \frac{q\left(X,(t-x)^{1 / 2}(t+x-2 X)^{1 / 2} \cos \theta,(t-x)^{1 / 2}(t+x-2 X)\right)^{1 / 2} \sin \theta}{\left((x-X)^{2}+(t-x)(t+x-2 X)\right)^{1 / 2}-(x-X)} \\
\times(t-x) d X d \theta .
\end{array}
$$

We want to see how the limit behaves if $t \rightarrow \infty, t-x \rightarrow 0$ and $t(t-x)=\eta$ is finite. Introducing the variable $\eta$, we have as limit

$$
\begin{gathered}
-\frac{1}{4 \pi} \int \frac{q(X, \sqrt{2 \eta} \cos \theta, \sqrt{2 \eta} \sin \theta) \eta t^{-1}}{(x-X)(1+\eta /(x-X))-(x-X)} d X d \theta \\
\rightarrow \frac{-1}{4 \pi} \int q(X, \sqrt{2 \eta} \cos \theta, \sqrt{2 \eta} \sin \theta) d X d \theta
\end{gathered}
$$

Hence, $F$ tends to a function of $\eta$. It has the right limit as $\eta \rightarrow 0$ and thus the linearized solution tends to a function of $\eta, x_{2}, x_{3}$.

For two space variables $x, y$ we have

$$
\begin{aligned}
P_{2 \text { scat }}=F & =\frac{1}{2 \pi} \int \frac{q\left(x^{\prime}, y^{\prime}\right) \delta\left(t^{\prime}-x^{\prime}\right)}{\left(\left(t-t^{\prime}\right)^{2}-2 x^{\prime}(t-x)-\left(y-y^{\prime}\right)^{2}\right)^{1 / 2}} \\
& =\frac{1}{2 \pi} \int \frac{q\left(x^{\prime}, y^{\prime}\right) d x^{\prime} d y^{\prime}}{\left(t^{2}-x^{2}-2 x^{\prime}(t-x)-\left(y-y^{\prime}\right)^{2}\right)^{1 / 2}} .
\end{aligned}
$$

Here the range of integration is over the domain where the square root is real.

Setting $y-y^{\prime}=\rho \cos \theta$ with $(t-x)\left(t+x-2 x^{\prime}\right)=\rho^{2}$, we have

$$
F=-\frac{1}{2 \pi} \int q\left(x^{\prime}, y-\rho \cos \theta\right) d \theta d x^{\prime} \text {. }
$$


And for large $t, \rho^{2} \rightarrow 2 \eta+\eta^{2} / x^{2}-2 x^{\prime} \eta / x$, where again $\eta=x(t-x)$, and thus

$$
F \rightarrow-\frac{1}{2 \pi} \int q\left(x^{\prime}, y-\sqrt{2 \eta} \cos \theta\right) d \theta d x^{\prime}=F(\eta, y)
$$

We proceed to get the solution as a solution of a simple partial differential equation. Let $V\left(\eta, x_{\perp}\right)$ be an approximate solution to the wave equation for $\eta=$ $\left(t-x_{1}\right)$ finite; then

$$
\left(2 t-x_{1}\right)^{2} V_{\eta \eta}+2 V_{\eta}-V_{\eta \eta} t^{2}-\Delta_{\perp} V=0
$$

where $\Delta_{\perp}$ is in the space orthogonal to $x_{1}$, or

$$
\left(\left(t+t^{-1} \eta\right)^{2}-t^{2}\right) V_{\eta \eta}+2 V_{\eta}-\Delta_{\perp} V=0
$$

or

$$
2 \eta V_{\eta \eta}+2 V_{\eta}-\Delta_{\perp} V+t^{-2} \eta^{2} V_{\eta \eta}=0
$$

We could find a series in powers of $t^{-2}$ but we look only at the leading term, which satisfies the equation

$$
2\left(\eta V_{\eta}\right)_{\eta}-\Delta_{\perp} V=0
$$

with the boundary condition $V(0)=-\frac{1}{2} \int_{-\infty}^{+\infty} q\left(x_{1}, x_{\perp}\right) d x_{1}$. The solution is unique. If we multiply by $V_{\eta}$ we have

$$
\left(\eta V_{\eta}^{2}\right)_{\eta}+V_{\eta}^{2}-\operatorname{div}_{\perp}\left(V_{\eta} \nabla_{\perp} V\right)+\frac{1}{2}\left|\nabla_{\perp} V\right|_{\eta}^{2}=0
$$

and if we integrate with respect to $x_{\perp}$ we have

$$
\frac{d}{d \eta}\left(\int\left(\eta V_{\eta}^{2}+\frac{1}{2}\left|\nabla_{\perp} V\right|^{2}\right) d x_{\perp}+\iint_{0}^{\eta} V_{\eta}^{2} d \eta d x_{\perp}\right)=0 .
$$

Hence, uniqueness and existence follow by standard projection methods. For any given $q$ we can easily find the solution in the boundary layer, and as $\eta \rightarrow \infty$ the identity (4.4) can be used to show $\int\left|V_{\eta}\right|^{2}\left|d x_{\perp}\right| \rightarrow 0$ faster than $1 / \eta$. However, solving the equation (4.3) backward to find $V\left(0, x_{\perp}\right)$ from $V\left(\eta, x_{\perp}\right)$ appears to be ill-posed, as the equation behaves like the heat equation as $\eta \rightarrow 0$.

5. Computing the Forward or Direct Problem and Application to the Back-Scattering Problem. In order ultimately to apply the algorithm of Section 3 , it is necessary to be able to compute the forward problem for $P_{\text {scat }}(e, t, x)$. This has been done in two ways.

A. The first way is to use a mesh with $\Delta t=\frac{1}{2} \Delta x_{1}=\frac{1}{2} \Delta x_{2}$. On the edge, $t=x_{1}$, as we can always rotate the variables so that $e=(1,0)$; we have the values $P_{\text {scat }}$ given by the boundary condition $P_{\text {scat }}=-\frac{1}{2} \int_{-\infty}^{x_{1}} q\left(s, x_{2}\right) d s,(1.4)$. $\left(\Delta / \Delta x_{1}, \Delta t / \Delta x_{2} \leq \sqrt{2} / 2\right.$ is the fundamental stability bound in two dimensions.)

We use the standard difference scheme for the operator $\square$ and we note in Figure 5.1 the circled points which cannot be reached in a step forward in time. They are 


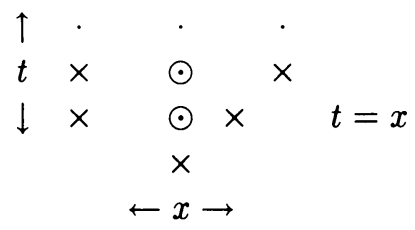

FIGURE 5.1

Stencil near the surface $t=x_{1}$.

obtained by taking a weighted mean with the points to the left and right (marked by $x$ ). The boundary layer eventually is not resolved by the mesh, and as one approaches this situation the computation develops an oscillation. We denote the approximate scattered wave solution by $w(x, y, t)$.

The scheme described above is stable and has been tested on a range of potentials. Attempts to find a stable fourth-order extension have been unsuccessful so far. To illustrate a sample computation, we consider potentials of the form

$$
\begin{aligned}
& q(x, y)=\varepsilon \alpha 2 x e^{-\alpha\left(x^{2}+y^{2}\right)}, \quad x \geq 0, \\
& q(x, y)=0, \quad x \leq 0 \text {. }
\end{aligned}
$$

In the computation we took $\alpha=5$, leading to a highly peaked potential.

In Figure 5.2 we plot $w(x, y, t)$ as a function of $x$ and $y$ for several values of $t$. Here, $\varepsilon=1$. It is apparent that as $t$ increases, the solution is composed of a smooth, propagating component and a nonpropagating steeply changing boundary layer, in agreement with the theory of the preceding section. For large values of $t$ the boundary layer is not well resolved and localized oscillations develop. The oscillations become more severe as $t$ increases due to the narrowing of the boundary layer. The grid size for the computations in Figure 5.2 is 0.04 .

In order to ascertain the effect of the lack of resolution of the boundary layer on the physically relevant cross section, we plot in Figure 5.3 the back-scattered cross section $\sqrt{r} w(l-r, 0, t)$ taken at $r=10$ for various grid sizes and for various values of $\varepsilon$. It can be seen that the cross section is smooth and is mesh converged even though oscillations are present in the boundary layer. For large $\varepsilon$ the breakdown is due to the fact that errors from mesh effects are as important as changes in $q$. We recollect that $q$ has the dimensions of (length) $)^{-2}$ so that $q^{-1 / 2}$ of the order of the mesh size is not a reasonable computation.

In order to elucidate the structure in the boundary layer, in Figure 5.4 we plot $w(x, 0, t)$ for $t=6,7,8,9,10$ for different grids and different values of $\varepsilon$. The results are presented only in a vicinity of the boundary layer. It is apparent that the oscillations are grid-dependent and are smaller as the grid is refined. The more important feature is that the scattered wave stays very much the same.

A sample computation using 801 points in the $x$ direction and 401 points in the $y$ direction and run for 14 units of time required $\sim 55 \mathrm{sec}$. on the Cray $1-\mathrm{S}$. In this case we had the mesh size $h=0.04$. No symmetry around $y=0$ is assumed, even though the solution is symmetric. A calculation with $h=0.08$ and using $N x=401$ and $N y=301$, which gave an accurate cross section, required $15 \mathrm{sec}$. We also ran on the Cray X-MP at the Naval Research Laboratory. 

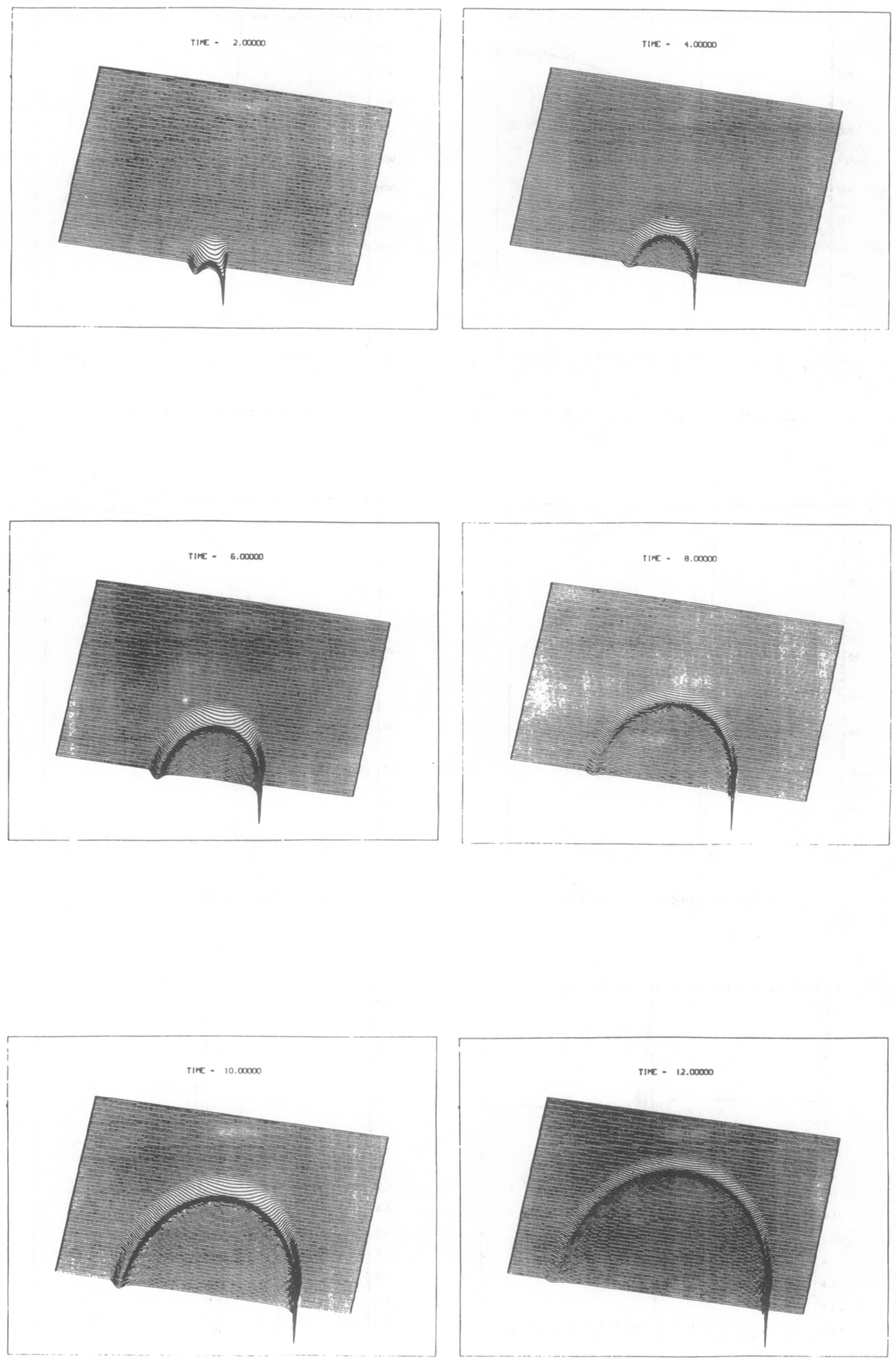

FIGURE 5.2

A time series of the scattered wave. The speed of propagation is 1 . 

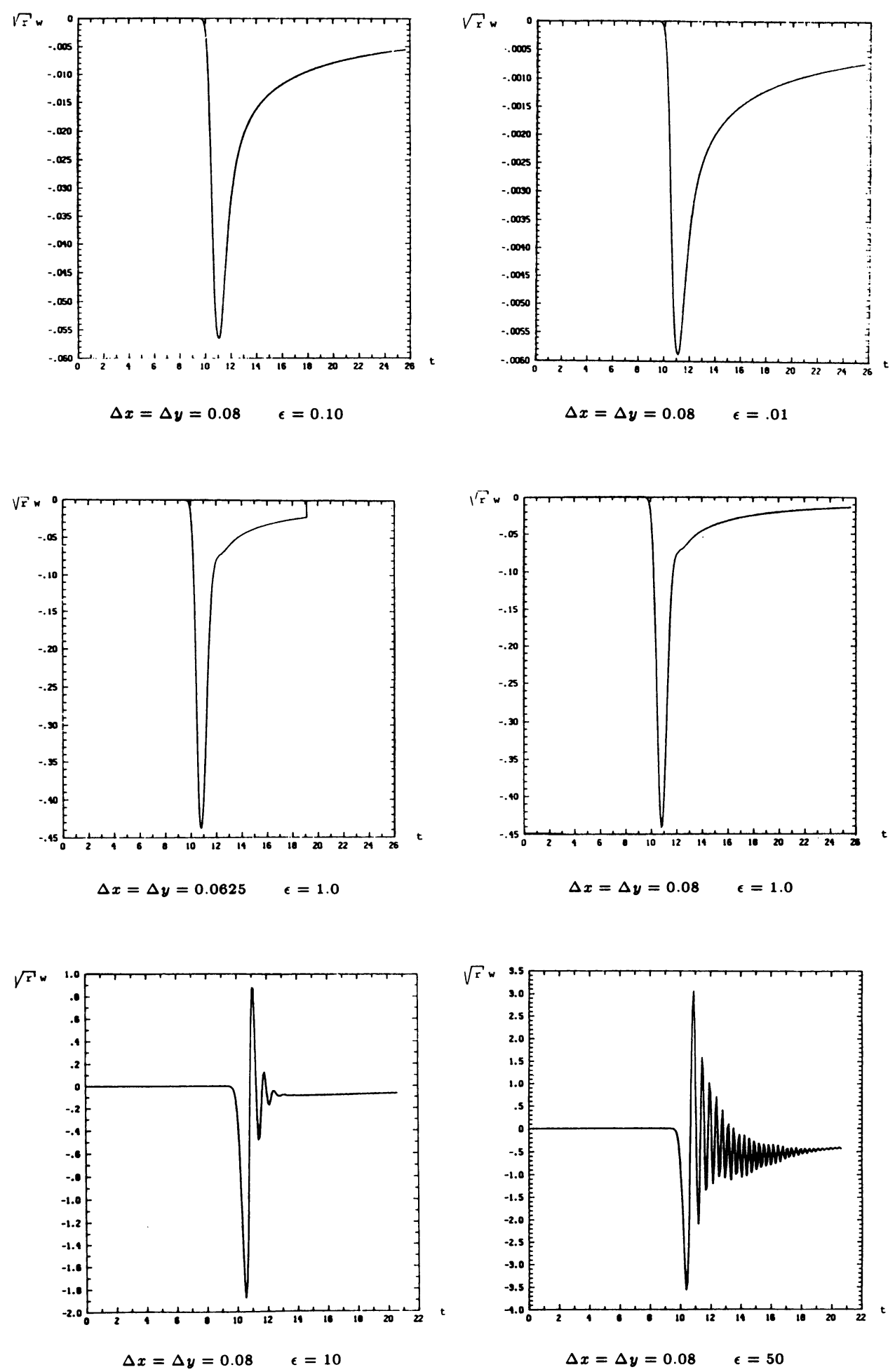

FIGURE 5.3

Back-scattered cross sections. 

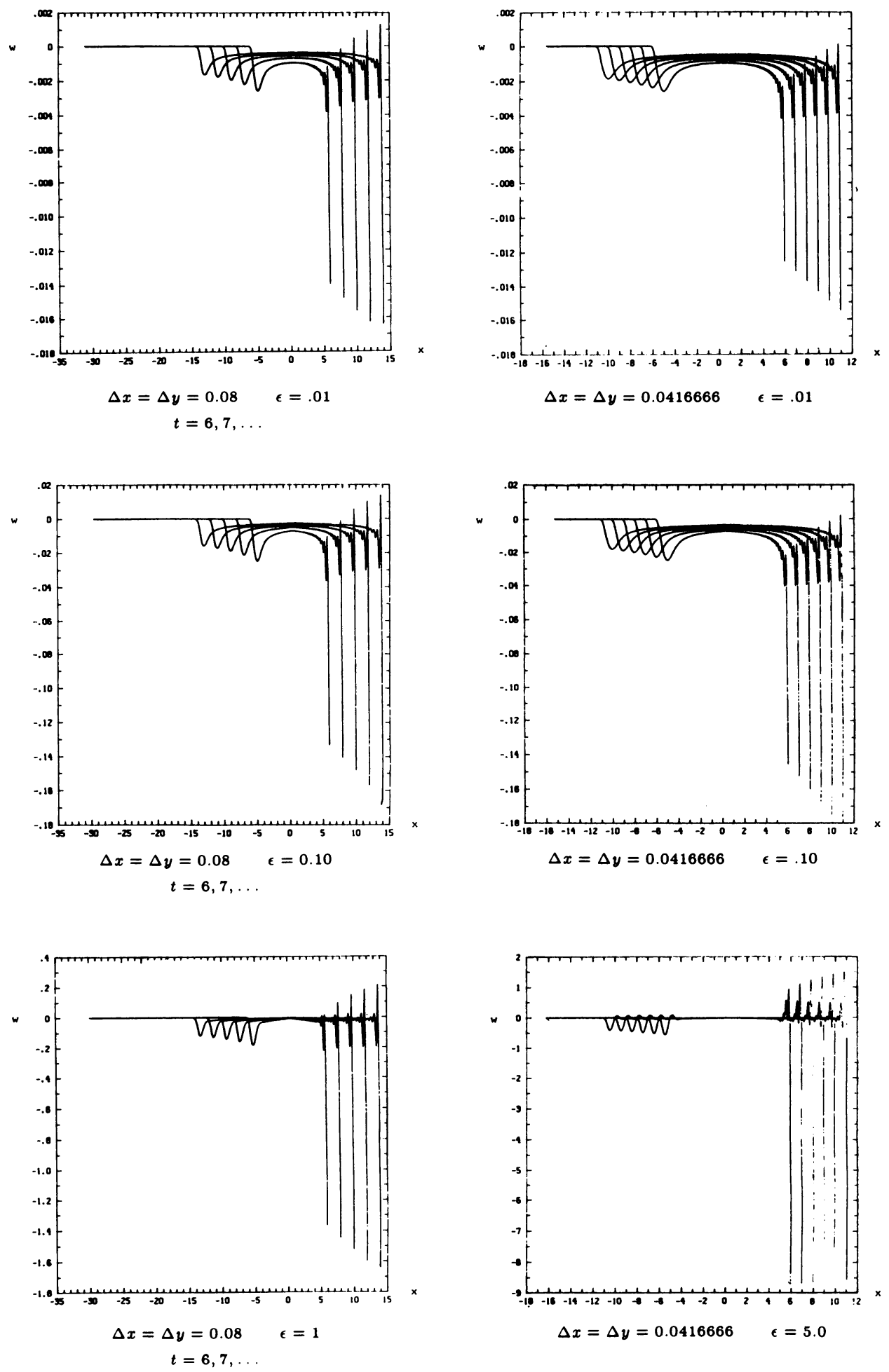

FIGURE 5.4

Structure of the boundary layer. 
The far field boundary conditions in $y$ are very sensitive near the plane $t=x$. The radiation condition $u \sim F(t-r, \theta) / \sqrt{r}$, when implemented numerically, proved unstable in all implementations near this plane. (It is incorrect.) The computations presented above were obtained using the boundary condition

$$
u \sim F(t-y, x)
$$

which proved stable. Very little reflections were observed for (5.1) provided the boundary included at least twice the support of $q$. We are presently attempting a correct radiation condition in a region bounded away from the boundary layer.

It is clear that no uniform grid will provide adequate resolution of the boundary layer for large $t$. An accurate computation of the boundary layer would require either a severely stretched mesh or the use of the boundary layer equations developed previously. Although the computations suggest that the far field can be accurately computed without resolving the boundary layer, because the oscillations do not propagate, it remains to be seen what effect these oscillations will have on the iteration scheme proposed above. We note that the functional $F(x ; q)$ does not involve data near the boundary layer for $q$ of compact support.

B. The second scheme used a Goursat algorithm for the variables $t-x_{1}, t+$ $x_{1}$. With a mesh size in $x_{2}$ that is twice the mesh size in $x_{1}, t$ the scheme was stable. Using only $50 x$-points, $30 y$-points, the program was run for $t \leq 3.0$. The computation with the coarse mesh size in $x_{1}$ equal to 0.1 gave similar results to the fine mesh for the case where $q$ is given by (5.1). The machine was a Cyber 180 .

To use either of these methods in the iterative algorithm based on (3.1), we have a very large integral to compute on each iteration, namely

$$
\iint_{e \cdot x^{\prime}<e \cdot x} P_{\mathrm{scat}}\left(e, e \cdot\left(2 x-x^{\prime}\right), x^{\prime}\right) q\left(x^{\prime}\right)\left|d x^{\prime}\right||d e| .
$$

The values of $P_{\text {scat }}$ are generated for each $e$, and we may rotate the variables and compute

$$
\int \tilde{P}_{\text {scat }}\left(e_{0}, 2 x_{1}-x_{1}^{\prime}, x^{\prime}\right) q\left(U x^{\prime}\right)\left|d x^{\prime}\right|
$$

with $U$ a unitary matrix that transforms $e$ into $e_{0}$ and $\tilde{P}_{\text {scat }}$ denoting the scattered wave from $q\left(U x^{\prime}\right)$. Then the order of computations is: for each $t$ compute values of $\tilde{P}$ for each fixed row $x_{1}^{\prime}=$ const running through the values of $x_{2}^{\prime}$. The product $\tilde{P} q\left|\Delta x^{\prime}\right|$ at each point is to be added for all $x_{2}^{\prime}$ and the sum is then accumulated at all values of $x_{1}=\frac{1}{2}\left(t+x_{1}^{\prime}\right)$. Since in method A, $t=j \Delta t$ and $x_{1}^{\prime}=2 m \Delta t$, $x_{1}=(m+j / 2) \Delta t$, this operation should be performed only at every other time step.

6. Other Relations for $q$ in Higher Dimensions. The basic relation of Section 1 is an integral relation for $q$ in terms of back-scattering data. However, there are other possible inverse problems. For example, suppose all the scattered field from $\delta\left(t-x_{1}\right)$, i.e., $S\left(e_{0}, t-|x|, e^{\prime}\right)$, is known; can we find $q(x)$ ? Here at least we can say that if $S \equiv 0$ then $q \equiv 0$, a conclusion we cannot draw from our basic relation. The method uses a generalization of the Deift-Trubowitz trace formula derived in the time-dependent framework. 
THEOREM. For $q$ of compact support,

$$
\Delta q=+4 \frac{\partial^{2}}{\partial x_{1}^{2}} \int_{-\infty}^{+\infty} \int P\left(-e_{0},-t, x\right) P\left(-e^{\prime}, s-t, x\right) S_{s}\left(e_{0}, s, e^{\prime}\right) d e^{\prime} d s d t
$$

with

$$
S\left(e_{0}, s, e^{\prime}\right)=c_{n} \int \mathbf{S}\left(e_{0}, s+\frac{1}{2} \tau^{2}, e^{\prime}\right) \tau^{n-2} d \tau,
$$

$c_{n}=c(n)$, where $S$ is the scattered field and $n$ is the number of space dimensions.

Remark. This formula can be transformed into the frequency domain and is easily seen to become

$$
\Delta q=4 \frac{\partial^{2}}{\partial x_{1}^{2}} \int \omega p^{2}\left(-e_{0}, \omega, x\right) \hat{\mathbf{S}}\left(e_{0}, \omega, e\right) d e^{\prime} d \omega
$$

where $p$ is the Fourier transform of $P$ and

$$
\hat{S}\left(e_{0}, \omega, e^{\prime}\right)=c_{n} \int_{0}^{\infty} e^{-i \omega t^{2}} \hat{\mathbf{S}}\left(e_{0}, \omega, e^{\prime}\right) t^{n-2} d t .
$$

Here, - denotes Fourier transform.

An iteration for finding $q$ from $\hat{S}$ could be done by using trial $q$ 's to find $p$ and determining a new $q$ from the above formula.

Proof of the Theorem. We obtain a preliminary formula using the two solutions

$$
P\left(e_{0}, t, x\right) \text { and } P\left(-e_{0},-t, x\right) .
$$

We would like to consider

$$
\int_{-\infty}^{+\infty} P_{t}\left(e_{0}, t, x\right) P\left(-e_{0},-t, x\right) d t
$$

but it does not exist. But we can form, as we shall see,

$$
\begin{aligned}
& \int_{-\infty}^{+\infty}\left(P_{t}\left(e_{0}, t, x\right) P_{x_{1}}\left(-e_{0},-t, x\right)+P_{x_{1}}\left(e_{0}, t, x\right) P_{s}\left(-e_{0},-t, x\right)\right) d t \\
& =\int_{-\infty}^{+\infty} \frac{\partial}{\partial x_{1}}\left(P_{t}\left(e_{0}, t, x\right) P\left(-e_{0},-t, x\right)\right) d t=I .
\end{aligned}
$$

We must extend (1.3) to more terms, and we find

$$
P\left(e_{0}, t, x\right)=\delta\left(t-x_{1}\right)-\frac{1}{2} \int_{-\infty}^{x_{1}} q\left(s, x_{2}\right) d s H\left(t-x_{1}\right)+B_{+}(x) C\left(t-x_{1}\right),
$$

where $\dot{C}=H$. On the other hand,

$$
P\left(-e_{0},-t, x\right)=\delta(t-x)+\frac{1}{2} \int_{\infty}^{x_{1}} q\left(s, x_{2}\right) d s\left(1-H\left(t-x_{1}\right)\right)+B_{-}(x) C^{*}(t-x),
$$

where $\dot{C}^{*}=1-H$.

The two solutions have common support only on $t=x_{1}$, so we can use the above expansions.

The product $\delta^{\prime}\left(t-x_{1}\right) \delta(t-x)$ integrates to zero. The cross term

$$
\begin{aligned}
\delta^{\prime}\left(t-x_{1}\right) & \left(\frac{1}{2} \int_{\infty}^{x_{1}} q\left(s, x_{2}\right) d s\right)\left(1-H\left(t-x_{1}\right)\right) \\
& -\frac{1}{2} \int_{-\infty}^{x_{1}} q\left(s, x_{2}\right) d s \delta\left(t-x_{1}\right) \delta\left(t-x_{1}\right),
\end{aligned}
$$


when integrated with respect to $t$, gives, when differentiated with respect to $x_{1}$,

$$
\int \frac{\partial}{\partial x_{1}}\left(-\frac{1}{2} \delta\left(t-x_{1}\right) \delta\left(t-x_{1}\right) \int_{-\infty}^{+\infty} q\left(s, x_{2}\right) d s\right) d t
$$

which vanishes. All these formulas can be established using limits of Gaussians instead of delta functions.

The remaining terms yield

$$
\begin{aligned}
\int_{-\infty}^{+\infty} & \frac{\partial}{\partial x_{1}}\left(\delta\left(t-x_{1}\right)\left(1-H\left(t-x_{1}\right)\right)\left(-\frac{1}{2} \int_{\infty}^{x_{1}} q\left(s, x_{2}\right) d s\right)\left(\frac{1}{2} \int_{-\infty}^{x_{1}} q\left(s, x_{2}\right) d s\right)\right. \\
& \left.+\delta^{\prime}\left(t-x_{1}\right) B_{-}(x) C^{*}\left(t-x_{1}\right)+\delta(t-x) B_{+}(x) H\left(t-x_{1}\right)\right) d t \\
= & -\frac{1}{8} \frac{\partial}{\partial x_{1}}\left(\int_{\infty}^{x_{1}} q\left(s, x_{2}\right) d s \int_{-\infty}^{x_{1}} q\left(s, x_{2}\right) d s\right)+\frac{\partial}{\partial x_{1}} \frac{1}{2}\left(-B_{-}+B_{+}\right) \\
= & -\frac{1}{8} q(x) \int_{-\infty}^{x_{1}} q\left(s, x_{2}\right) d s-\frac{1}{8} q(x) \int_{\infty}^{x_{1}} q\left(s, x_{2}\right) d s \\
& +\frac{1}{2} \frac{\partial}{\partial x_{1}}\left(-B_{-}+B_{+}\right)
\end{aligned}
$$

or

$$
I=-\frac{1}{16} \frac{\partial}{\partial x}\left(\left(\int_{-\infty}^{x_{1}} q\left(s, x_{2}\right) d s\right)^{2}+\left(\int_{\infty}^{x_{1}} q\left(s, x_{2}\right) d s\right)^{2}\right)+\frac{1}{2} \frac{\partial}{\partial x_{1}}\left(-B_{-}+B_{+}\right) .
$$

We next compute $B_{ \pm}$. We have, by standard theory,

$$
\begin{aligned}
& 2 B_{+x_{1}}+\frac{1}{2} \Delta \int_{-\infty}^{x_{1}} q\left(s, x_{2}\right) d s+q\left(-\frac{1}{2} \int_{-\infty}^{x_{1}} q\left(s, x_{2}\right) d s\right)=0 \\
& 2 B_{-x_{1}}-\frac{1}{2} \Delta \int_{+\infty}^{x_{1}} q\left(s, x_{2}\right) d s+q\left(+\frac{1}{2} \int_{-\infty}^{x_{1}} q\left(s, x_{2}\right) d s\right)=0
\end{aligned}
$$

Hence,

$$
\begin{gathered}
2 \frac{\partial^{2}}{\partial x_{1}^{2}}\left(B_{+}-B_{-}\right)+\Delta q-\frac{1}{4} \frac{\partial^{2}}{\partial x_{1}^{2}}\left(\int_{-\infty}^{x_{1}} q\left(s, x_{2}\right) d s\right)^{2} \\
-\frac{1}{4} \frac{\partial^{2}}{\partial x_{1}^{2}}\left(\int_{-\infty}^{x_{1}} q\left(s, x_{2}\right) d s\right)=0 .
\end{gathered}
$$

Thus we have from (6.2), $\partial I / \partial x_{1}=-\frac{1}{4} \Delta q$ or

$$
\Delta q=-4 \int_{-\infty}^{+\infty} \frac{\partial^{2}}{\partial x_{1}^{2}}\left(P_{t}\left(e_{0}, t, x\right) P\left(-e_{0}, t, x\right)\right) d t
$$

This is similar to a preliminary formula of Deift and Trubowitz [3] but we note that the Fourier transform of $P$ is not the Jost function. That solution would have to behave like $\delta\left(t-x_{1}\right)$ as $x_{1} \rightarrow-\infty$, and in higher than one space dimension this solution does not exist in general.

We next relate $P(e, t, x)$ to its scattered data $S\left(e, s, e^{\prime}\right)$. We define $\mathbf{S}$ by

$$
S\left(e, s, e^{\prime}\right)=c_{n} \int_{0}^{\infty} \mathbf{S}\left(e, s+\frac{1}{2} \tau^{2}, e^{\prime}\right) \tau^{n-2} d \tau
$$

for $n \geq 2$. 
Here, $c_{n}$ depends only on the dimension. To determine $\mathbf{S}$ from $S$ for $n=3$ is a simple integration. For $n>3$ we differentiate with respect to $s$ several times, and then the exponent in $\tau$ can be reduced to 1 since $\mathbf{S}_{s}=\tau \mathbf{S}_{\tau}$ and the right-hand side can be integrated. For $n=2$ the transform is an Abel transform and can be inverted uniquely.

Then we have the following

LEMMA. If $S$ is given by (6.5), then for $t \geq e \cdot x$,

$$
P(e, t, x)=P(-e, t, x)+\int P\left(-e^{\prime}, s-t, s\right) S\left(e, s, e^{\prime}\right) d e^{\prime} d s .
$$

Proof. The right-hand side is a solution of the differential equation since it is an integral over solutions. We are assuming here that $S$ decays sufficiently rapidly when its middle argument goes to infinity. Let $t$ be sufficiently large so that on the range of $s$ where $S$ is not, say, less than $\varepsilon, P\left(-e^{\prime}, s-t, x\right)=\delta\left(s-t+e^{\prime} \cdot x\right)$. Then the right-hand integral is

$$
\begin{aligned}
\int \delta( & \left.-t+e^{\prime} \cdot x\right) S\left(e, s, e^{\prime}\right) d s d e^{\prime}+O(\varepsilon) \\
& =\int S\left(e, t-e^{\prime} \cdot x, e^{\prime}\right) d e^{\prime}+O(\varepsilon) \\
& =\int S\left(e, t-|x|+|x|\left(1-e^{\prime} \cdot x /|x|\right), e^{\prime}\right) d e^{\prime}+O(\varepsilon) \\
& =\int S\left(e, t-|x|+|x| 2 \sin ^{2} \frac{\theta}{2}, e^{\prime}\right) d e^{\prime}+O(\varepsilon),
\end{aligned}
$$

where $e^{\prime} \cdot x /|x|=\cos \theta$.

The leading term vanishes as $|x| \rightarrow \infty, t-|x|$ bounded, except near $\theta=0$. Using the methods used in proving our first basic relation, we find that the right-hand side tends as $|x| \rightarrow \infty$ and $\varepsilon \rightarrow 0$ to

$$
|x|^{-(n-1) / 2} c_{n} \int \mathbf{S}\left(e, t-|x|+\frac{1}{2} \tau^{2}, \frac{x}{|x|}\right) \tau^{n-2} d \tau,
$$

which by the definition of $\mathbf{S}$ is $|x|^{-(n-1) / 2} S(e, t-|x|, x /|x|)$. Hence the right-hand interval behaves for $t>e \cdot x$ like $P_{\text {scat }}(e, t, x)$ as $t \rightarrow \infty,(1.3)$. This uniquely determines $P_{\text {scat }}(e, t, x)$ for $t \geq e \cdot x$ by domain of dependence arguments extending to infinity.

Remark. It follows that $P_{\text {scat }} \equiv 0$ if $S$ or $\mathbf{S}\left(e, s, e^{\prime}\right) \equiv 0$ and hence, by (1.3), $q \equiv 0$. This is related to Marchenko's formulas, see [2].

We then have

$$
q=\int 2\left(P_{s}-P_{x_{1}}\right)\left(-e^{\prime}, s-x_{1}, x\right) \mathbf{S}\left(e, s, e^{\prime}\right) d s d e^{\prime} .
$$

The same kind of dependence arguments show, since

$$
P(-e,-t, x)=\int P\left(e^{\prime}, s-t, x\right) \delta(s) \delta\left(e+e^{\prime}\right) d e d s^{\prime},
$$

that as $t \rightarrow+\infty$

$$
P(-e,-t, x)+\int P\left(e^{\prime}, s-t, x\right) \mathbf{S}\left(e, s, e^{\prime}\right) d s d e^{\prime}
$$

has the same data as $P(e, t, x k)$. Hence it is $P(e, t, x)$. 
Note that the boundary layer described in Section 3 is not a part of the data at $\infty$.

The analogue of the Deift-Trubowitz formula is obtained by substituting the $t$-derivatives of

$$
P(e, t, x)=P(-e,-t, x)+\int P^{\prime}\left(-e^{\prime}, s-t, x\right) \mathbf{S}\left(e, s, e^{\prime}\right) d e^{\prime} d s
$$

in (6.3) with $e=e_{0}$. Since $\int P P_{t} d t=0$ and the remaining terms can be integrated by parts with respect to $s$, we have completed the proof of the theorem.

Department of Engineering Sciences and Applied Mathematics

Northwestern University

Evanston, Illinois 60208

Department of Mathematics

Princeton University

Princeton, New Jersey 08544

Courant Institute of Mathematical Sciences

New York University

251 Mercer Street

New York, New York 10012

E-mail: Morawetz@ACF1.NYU.EDU

1. K. B. BUBE \& R. BURRIDGE, "The one-dimensional inverse problem of reflection seismology," SIAM Rev., v. 25, 1983, pp. 497-559.

2. R. BuRRIDGE, "The Gel'fand-Levitan, the Marchenko, and the Gopinath-Sondhi integral equations of inverse scattering theory, regarded in the context of inverse impulse-response problems," Wave Motion, v. 2, 1980, pp. 305-323.

3. P. Deift \& E. TRUBOWITZ, "Inverse scattering on the line", Comm. Pure Appl. Math., v. 32, 1979, pp. 121-251.

4. L. D. FADEEV, "Inverse problems of quantum scattering theory. II," J. Soviet Math., v. 5, 1976, pp. 334-396.

5. Roger G. NEWton, "Inverse scattering, Three dimensions II and III," J. Math. Phys., v. 21, 1980, pp. 1698-1715; v. 22, 1981, pp. 2191-2200. Also Erratum, v. 22, 1981, p. 631.

6. G. A. KRIEGSMANN \& C. S. MORAWETz, "The calculations of an inverse potential problems," SIAM J. Appl. Math., v. 43, 1983, pp. 844-854.

7. R. T. PROSSER, "Formal solutions of inverse scattering problems. I-IV," J. Math. Phys., v. 10,1969 , pp. $1819-1822$; v. 17,1976 , pp. $1775-1779$; v. 21, 1980, pp. 2648-2653; v. 23, 1982, pp. 2127-2130.

8. D. C. Stickler, "Inverse scattering in a stratified medium," J. Acoust. Soc. Amer., v. 74, 1983, pp. 994-1005. 